\title{
Elderly Perception of Critical Issues of Pedestrian Paths
}

\author{
Giulia Pulvirenti, Natalia Distefano, Salvatore Leonardi *
}

Department of Civil Engineering and Architecture, University of Catania, Italy

Received November 12, 2019 ; Revised Revised January 4, 2020; Accepted January 13, 2020

Copyright $\odot 2020$ by authors, all rights reserved. Authors agree that this article remains permanently open access under the terms of the Creative Commons Attribution License 4.0 International License

\begin{abstract}
Pedestrians aged over 65 are known to be a critical group in terms of road safety because they represent the age group with the highest number of fatalities or injured persons in road accidents. It is therefore important to identify and characterize how old pedestrians perceive pedestrian paths with respect to their age related declines in perceptual and physical abilities and with respect to their experiences as road users. The aim of this study is first of all to understand which critical issues old pedestrians found in the pedestrian paths they usually walk. More specifically, the final aim is to capture and analyze the key components that influence the elderly pedestrians' perception of pedestrian paths and to identify how these perceptions change for different pedestrian "profiles" based on human factors. The aspects related to human factors considered are the gender, the factors associated with the experience as road users and the factors related to age related problems (mobility, vision and hearing problems). The results show that the judgment expressed by the elderly on the critical issues of pedestrian paths they usually walk is significantly linked to gender, to their experience as road users, and to vision problem, which compromise the correct perception of the road environment. This is important to determine interventions and could support traffic engineers, planners, and decision-makers to consider the contributing factors in engineering countermeasures.
\end{abstract}

Keywords K-Means Cluster Analysis, Hierarchical Cluster Analysis, Survey, Road Safety, Vulnerable Users, Human Factor

\section{Introduction}

Deaths and injuries resulting from road traffic crashes is a serious problem globally and current trends suggest that this will continue to be the case in the foreseeable future [1]. According to the World Health Organization, the number of annual road traffic deaths reached 1.35 million in 2018, which is considered to be the eighth leading cause of death globally [2].

Of the total 1.35 million people dying in road traffic crashes annually, at least $30 \%$ are in urban areas [2]. Pedestrians are considered the most fragile road users in the transport system. They are at maximum risk compared to any other road users because of their fragility, slow pace, and their absence of protection [3]. In Europe, the safety of a pedestrian has been problematic for a long time. The actions taken to reduce pedestrian crashes have been much less notable compared to those for the total traffic accidents, although the total number of fatalities has decreased significantly during the period 2006-2016. In the European Union, a total of 5320 pedestrians were killed in road accidents in 2016, 21\% of all road fatalities [4].

The reality of an aging population, particularly in "economically developed" countries, has made the everyday mobility of seniors an issue of growing interest. In a context where an increasing number of people globally are dependent on cars, efforts to encourage walking - both utilitarian and leisurely - has become a public health priority [13]. However, public transportation and walking environments are not always well-adapted to accommodate the elderly. In order for walking to become an attractive, efficient, and safe mode of transportation for the elderly, the way public spaces are designed must be rethought/reconsidered in order to accommodate to their needs and preferences.

Age related declines in perceptual, cognitive, and physical abilities have been shown to result in non-optimal street-crossing decisions and behaviors $[6,7,8]$ and may contribute to the high rate of fatal or serious-injury crashes found for old pedestrians [9]. Because of age-related perceptual, cognitive, and motor limitations, and in line with [10] as well as [11], old pedestrians are expected to experience more difficulty than young pedestrians.

Considering the above facts, it is important to identify and characterize how old pedestrians perceive pedestrian paths with respect to their age related declines in perceptual and physical abilities and with respect to their experiences as road users.

The final aim of this study is first of all to understand 
which critical issues old pedestrians found in the pedestrian paths they usually walk. Moreover, this study seeks to analyze how old pedestrians' age related declines in perceptual and physical abilities (vision, hearing and mobility problems) and experiences as road user (no driving license, no still driving, accidents driving, accident pedestrian) can affect their opinion on the critical issues of pedestrian paths.

This is important to determine interventions and could support traffic engineers, planners, and decision-makers to consider the contributing factors in engineering countermeasures.

\section{An Overview of the Literature}

Walking is particularly important for the elderly, who are less likely than younger adults and children to participate in more vigorous forms of physical activity, more likely to experience social isolation and less likely to drive a car. Walking is also highly valued by seniors for a range of reasons, including improved health, wellbeing, independence, personal mobility and social connectedness. Moreover, walking is critical to allow older people to conduct day-to-day activities, such as shopping, attending meeting places (sporting clubs, libraries and community centers) and visiting essential services like doctors and hospitals [12]. In addition, in Italy 33\% of grandparents take care of grandsons every day [13]. This makes increasingly important to create safe walking opportunities around schools, as the two most vulnerable road user types walks together [14]. Walking can also reduce transport-related costs, including lower personal expenditure on fuel and vehicle maintenance. These aspects can be critical for older adults, who generally have lower annual incomes and for whom transport costs may represent a larger component of their expenses. The combination of these factors can result in transport disadvantage and social exclusion, which has been identified as a significant problem facing older adults [15].

It is well understood that walking has significant physical health benefits for older people, including reduced risk of many chronic diseases such as obesity, heart disease and diabetes [12, 15]. A well-established finding in the literature is a link between physical activity and decreased risk of many chronic diseases [16], including cardiovascular disease in people of all ages [17]. When analyzing the vulnerability of older pedestrians, it is important to consider the impact of physiological and cognitive changes that occur as people age. The World Health Organization in its 2013 report "Pedestrian Safety A Road Safety Manual for Decision Makers and Practitioners" states that the combination of the following factors increases the vulnerability of older pedestrians: deterioration in visual acuity results in older pedestrians accepting significantly smaller gaps in traffic than required when crossing roads; cognitive decline results in reduced ability to make safe judgments about walking speed and traffic gaps; reduced mobility results in an inability to react quickly and avoid crashes; frailty and existing health conditions can result in greater injury severity when a crash does occur; slower walking speeds can result in older pedestrians being stranded in the middle of the road when attempting to cross at signalized crossings.

Ageing results in gradual deterioration of agility (walking speed and balance), sensory perception (vision and hearing) and cognitive skills (attention and information processing speed). Older pedestrians can thus experience problems in situations that demand efficient cognitive processing, fast responses and quick actions [18].

Age-related changes reduce people's ability to undertake the many cognitive tasks required when crossing roads, such as finding a place to cross a road, looking for traffic, perceiving traffic, judging vehicle speeds and available gaps, deciding when to cross and then crossing the road. Older pedestrians are over-represented in crashes at complex intersections (particularly those with two-way traffic and/or multiple lanes) and when traffic volumes and speeds are high.

Outdoor walking, as a type of physical activity, takes place in outdoor spaces. It has well-known benefits for health in later life and older adults are recommended to take outdoor walks [19-21].

Evidence indicates that neighborhood safety, pedestrian infrastructure and aesthetics are important for supporting and encouraging outdoor walking [22-26] because pedestrians move slowly in outdoor spaces thus affording the ability to notice route characteristics [27].

These built environment attributes seem especially important for older adults' outdoor walking [19, 28, 29, 30]. For example, older adults may avoid walking to available attractive destinations located in walking distances due to high risk of accident [29]. Evidence has shown that for older adults' outdoor walking, maximizing the neighborhood aesthetics or quality of pedestrian infrastructure is more important than minimizing the distance to a destination [29].

Zandieh et al. [31] examine inequalities in perceived built environment attributes (i.e., safety, pedestrian infrastructure and aesthetics) and their possible influences on disparities in older adults' outdoor walking levels in low- and high-deprivation areas of Birmingham, United Kingdom. It applied a mixed-method approach, included 173 participants (65 years and over), used GPS technology to measure outdoor walking levels, used questionnaires and conducted walking interviews to collect data on perceived neighborhood built environment attributes. The results show inequalities in perceived neighborhood safety, pedestrian infrastructure and aesthetics in high- versus low-deprivation areas and demonstrate that they may influence disparities in participants' outdoor walking levels. Improvements of perceived neighborhood safety, 
pedestrian infrastructure and aesthetic in high-deprivation areas are encouraged. Most participants, particularly in high-deprivation areas, also talked about perceived uneven pavements, broken slabs, presence of potholes, cracks and obstacles (e.g., knocked down bollards) in pavements.

Kahlert and Schlicht [32] use an experimental study design with computer-simulated living environments to investigate the effect of micro-scale environmental factors (parking spaces and green verges with trees) on older people's perceptions of both motivational antecedents (dependent variables). Seventy-four consecutively recruited older people were randomly assigned watching one of two scenarios (independent variable) on a computer screen. The scenarios simulated a stroll on a sidewalk, as it is 'typical' for a German city. In version "A" the subjects take a fictive walk on a sidewalk where a number of cars are parked partially on it. In version "B", cars are in parking spaces separated from the sidewalk by grass verges and trees. Subjects assessed their impressions of both dependent variables. A multivariate analysis of covariance showed that subjects' ratings on perceived traffic safety and pedestrian friendliness were higher for version " $\mathrm{B}$ " compared to version " $\mathrm{A}$ ". The study suggests that elements of the built environment might affect motivational antecedents of older people's walking behavior.

Many researches examine the perception of elderly pedestrians about the quality and risks of the elements that characterize pedestrian routes (e. g., sidewalks and pedestrian crossings) in various investigation contexts. In particular, Lord et al. [33] examines the case of road crossings in the context of Montréal, Québec, Canada. The analyze are based on observations and questionnaires in order to bring to light a better understanding of the relationship between the crossing behaviors, characteristics and perceptions of the elderly. Five profiles of elderly people in both urban and suburban environments were established. A sample of 181 elderly pedestrians (65-93 years of age) were surveyed using a questionnaire. In addition to close-ended questions, respondents were asked to evaluate 17 environmental ambiance and risk behaviors according to various scales. Using principal component analysis (PCA) and hierarchical cluster analysis (HCA), the data was grouped into 6 categories that define and distinguish 7 profiles of elderly people. These profiles were explored according to the socioeconomic status and crossing behaviors of respondents. The probabilities of adopting different crossing behaviors were tested by employing logistic regression models. The results reveal greater variability in the perceptions of the elderly in terms of risk related to crossing behaviors and type of signalization at intersections.

Noh et al. [34] have investigated common and diverse contributory factors to elderly pedestrian injuries, by segmenting the elderly into the younger-old (between 65 and 74 years) and older-old (over 75 years). By employing single and interaction binary logit models, the study identified common risk factors for both elderly groups, as well as those that are particularly hazardous to the older-old. It was found that older age was the most critical risk factor leading to severe injury. A set of common contributory factors for both elderly groups was identified, including near overpass crossing, roadside, drunk, and truck. On the other hand, uphill, downhill, nighttime, and sidewalk were found to be a much higher risk to the older-olds.

Finally, a 2012 Belgian research [35] is particularly interesting for the purposes of this study. Van Cauwenberg et al. [35] sought to uncover the perceived environmental influences by elderly Flemish pedestrians. To get detailed and context-sensitive environmental information, it used walk-along interviews. Almost all participants mentioned the importance of the presence and quality of sidewalks. In case of absence of a sidewalk, characteristics of the streets and their shoulders were discussed. Streets with busy traffic or an uneven surface were perceived as less attractive to walk on. When a shoulder was present to walk on, uneven or muddy surfaces were disliked as well. When sidewalks were present, almost all participants mentioned issues related to the sidewalks' quality. They said they liked sidewalks that were well-maintained and even, and judged as hazardous and thus disliked cracked or uneven sidewalks, or sidewalks that had puddles, ice, snow, mud, or leaves. They also viewed sidewalks with steep cross-slopes as hazardous of becoming slippery during snowy and icy conditions. Adequate street lighting was mentioned as important for identifying fall hazards during walks after dark. Sidewalk width was also discussed. Participants preferred sidewalks wide enough for people to walk next to each other, to easily pass with a wheelchair and to maintain a safe distance from cars. To them, width means usable or walkable width. Walkable width narrows when a sidewalk has construction, parked cars, unkempt greenery and utility or light poles on it, all of which evoked negative responses. Separation of the sidewalk from motorized traffic by parked cars, bollards or vegetation was perceived as positive. Lastly, they said they disliked sidewalks that had high ramps to get on or off, slopes or stairs, because these elements increased the difficulty of walking. The presence of safe crossings was mentioned by some participants. Zebra crossings, supplemented with traffic lights in busy streets, were considered necessary to be able to cross streets safely. Participants reported to deviate from their shortest route in order to use a zebra crossing or traffic light to safely cross the street. Some participants expressed safety concerns related to the behaviors of other road users. Participants liked streets with slow traffic and disliked streets with speeding cars. This topic was mostly discussed near street crossings, especially when approaching cars were not visible (e.g. near sharp turns). Participants proposed solutions like speed bumps and chicanes to slow down traffic. On the other hand, participants also mentioned car drivers being 
very courteous and giving priority to pedestrians at crossings. Not only speeding cars were disliked but careless cyclists on sidewalks were mentioned as dangerous as well.

\section{Methods}

\subsection{Participants and Questionnaire}

In order to investigate which critical issues old pedestrians found in the pedestrian paths they usually walk and how their age related declines in perceptual and physical abilities and their experiences as road user can affect the opinion on the critical issues of pedestrian paths, a survey was developed.

The investigation techniques based on surveys represent a very effective tool for the study of lot of issues of transport interest [36-41]. These techniques especially become indispensable when it is not possible to evaluate through experimental investigations the indicators associated with the subjective judgments of different road users.

A 22 items questionnaire was used to collect the participants' opinions. The questionnaire was divided into the following 5 sections:

- $\quad$ Section 1: participants reported their age, their gender and other basic socio-demographic characteristics information in the first section;

- $\quad$ Section 2: this section included questions regarding the experience as road users of participants. Participants were asked if they ever had the driving license, if they still drove, if they ever had accidents while driving and if they ever had accidents as pedestrians;

- $\quad$ Section 3: the third section contained questions about the age related declines of perceptual and physical abilities. Participants were asked if they had vision problems, hearing problems and mobility problems.

- $\quad$ Section 4: this section consisted of an open-ended question related to the critical issues of pedestrian paths. Participants could express freely their opinion related to the critical issues and the problems they found in the pedestrian paths they usually walked.

- $\quad$ Section 5: this section consisted of an open-ended question related to the solutions for critical issues of pedestrian paths. Participants could express freely their opinion related to the solutions they thought could improve the safety of pedestrian paths they usually walked.

The questionnaire underwent thorough piloting and revision, through 20 interviews face to face. This was done to ensure the suitability of the questions for the target people and to assess the acceptability of the wording, as well as the understanding of the questions.

Since the aim of this study was to explore the perception of old pedestrians of the critical issues of pedestrian paths, this study focuses on the first four sections of the questionnaire.

The survey was conducted in 5 different locations in Catania, Italy. The locations were specifically chosen near to attraction poles for old pedestrians (e.g. centers for the elderly, squares, churches).

Participants were recruited in person, so as to select exclusively people over 70. Participants were briefed of the nature and time required to participate in the study prior to commencement. After their consent was obtained, the questionnaire started. It was decided to question directly the participants, instead of leaving them alone with the questionnaire, in order to provide visual aids and detailed explanations and clarifications. Each survey lasted approximatively 20 minutes. Participants were assured of anonymity and confidentiality.

The total sample comprised 322 participants (164 men and 158 women). Participants who didn't complete the questionnaire or who gave uncertain answers were excluded. The respondents excluded were about $5 \%$ of the sample.

The final sample was composed by 306 participants (156 men and 150 women). The majority of respondents (50.33\%) were aged between 70 and 75 . 28.10\% of respondents were aged between 75 and 80 and $21.57 \%$ of respondents were over 80 .

\subsection{Analytical Method}

In order to analyze the survey data a cluster analysis was carried out. Cluster analysis is a multivariate data exploration method. The primary objective of this analysis is to identify groups or "clusters" based on the similarities between the data points or a "natural” grouping. This can be done with a single data point or a combination of data points of interest such a series of questionnaires. There are several ways to perform a cluster analysis, but the two primary methods are K-Means and Hierarchical.

K-Means clustering is the most commonly used unsupervised machine learning algorithm for partitioning a given data set into a set of $\mathrm{k}$ groups (i.e. k clusters), where $\mathrm{k}$ represents the number of groups pre-specified by the analyst. It classifies objects in multiple groups (i.e., clusters), such that objects within the same cluster are as similar as possible (i.e., high intra-class similarity), whereas objects from different clusters are as dissimilar as possible (i.e., low inter-class similarity). In K-Means clustering, each cluster is represented by its center (i.e., centroid) which corresponds to the mean of points assigned to the cluster.

The first step when using K-Means clustering is to indicate the number of clusters $(\mathrm{k})$ that will be generated in the final solution. The algorithm starts by randomly selecting $\mathrm{k}$ objects from the data set to serve as the initial centers for the clusters. The selected objects are also known 
as cluster means or centroids. Next, each of the remaining objects is assigned to its closest centroid, where closest is defined using the Euclidean distance between the object and the cluster mean.

This step is called "cluster assignment step". After the assignment step, the algorithm computes the new mean value of each cluster. The term cluster "centroid update" is used to design this step. Now that the centers have been recalculated, every observation is checked again to see if it might be closer to a different cluster. All the objects are reassigned again using the updated cluster means. The cluster assignment and centroid update steps are iteratively repeated until the cluster assignments stop changing (i.e. until convergence is achieved). That is, the clusters formed in the current iteration are the same as those obtained in the previous iteration.

The basic idea behind K-Means clustering consists of defining clusters so that the total intra-cluster variation (known as total within-cluster variation) is minimized. There are several K-Means algorithms available. The standard algorithm is the Hartigan-Wong algorithm (1979), which defines the within-cluster variation as the sum of squared distances Euclidean distances between items and the corresponding centroid:

$$
\mathrm{W}\left(\mathrm{C}_{\mathrm{k}}\right)=\sum_{x_{i} \in C_{k}}\left(x_{i}-\mu_{k}\right)^{2}
$$

where:

- $\mathrm{W}(\mathrm{Ck})=$ total within-cluster variation

- $\quad \mathrm{xi}=$ a data point belonging to the cluster $\mathrm{Ck}$

- $\mu_{\mathrm{k}}=$ the mean value of the points assigned to the cluster $\mathrm{Ck}$

Each observation (xi) is assigned to a given cluster such that the sum of squares (SS) distance of the observation to their assigned cluster centers $\left(\mu_{\mathrm{k}}\right)$ is minimized.

So, the final goal of K-Means is to minimize the total within-cluster sum of square.

$$
\begin{gathered}
\text { Tot. within - cluster }=\sum_{k=1}^{k} \mathrm{~W}\left(\mathrm{C}_{\mathrm{k}}\right) \\
=\sum_{k=1}^{k} \sum_{x_{i} \in C_{k}}\left(x_{i}-\mu_{k}\right)^{2}
\end{gathered}
$$

This quantity also measures the compactness (i.e. goodness) of the clustering.

The second approach to a cluster analysis is the Hierarchical method. In contrast to K-Means, in the hierarchical method clusters are merged based on distance from each other. The method considers each data point as its own individual data point and then clusters data points based on the distance between each data point. At first, each data point is grouped with the data point closest to it as defined by one of the linkage methods for hierarchical clustering defined below:
- $\quad$ Single Linkage: the distance between the closest data points of the two clusters.

- Complete Linkage: the distance between the data points of the two clusters which are the farthest apart from each other.

- Average Linkage: comparing between all pairs and averages of all distances. Also called UPGMA Unweighted Pair Group Mean Averaging.

- Centroid Method: finding the mean vector location for each of the clusters and taking the distance between the two centroids.

- Ward's Method: Uses statistical analysis methods such as error sum of squares and R-squared to determine groupings of data points.

Then, each of these groups is merged with the groups closest to its group mean, and so on. This continues until all groups have been merged.

The optimal number of clusters with the Hierarchical method is determined by the minimum number of groups with the maximum amount of distance between group means. Frequently, this is illustrated with a dendrogram of the merging clusters.

Using a dendrogram, the ideal number of clusters is determined by the number of clusters intersected when drawing a vertical line through the largest horizontal distance between merging clusters.

\subsection{Model development}

Cluster analysis was used in this study in order to explore the safety perceptions of elderly pedestrians. Starting from the results of the survey, cluster analysis was developed to answer the following research questions:

1. Can we group together old pedestrians with a similar perception of critical issues of pedestrian paths?

2. How can we interpret the groups obtained? What do old pedestrians belonging to the same group have in common?

3. Which variables do mostly affect the determination of the groups?

The nominal variable considered is "critical issues of pedestrian paths", with the sixteen possible items showed in Table 1. These items were deduced from the open-ended question related to the critical issues of pedestrian paths of Section 4 of the questionnaire.

The 8 variables considered are instead showed in Table 2 . The variable No driving license indicates whether the respondents had not ever got the driver license, that means whether the respondents had not ever drove. The variable No still driving indicates whether the respondents were not still driving when they answered the questionnaire. The variable Accidents driving indicates whether the respondents had ever had an accident when they were driving. The variable Accidents pedestrian indicates whether the respondents were ever hit by a car (or another 
vehicle) when they were walking. The variables Vision problems, Hearing problems and Mobility problems indicates whether the respondents have vision, hearing or mobility problems respectively. These variables are therefore representative of the respondents' age related declines in perceptual and physical abilities of respondents. Finally, the variable Gender of respondents was included in the analysis.

Table 1. Nominal variable: critical issues of pedestrian paths

\begin{tabular}{|c|l|}
\hline \multicolumn{2}{|l|}{ Critical issues of pedestrian paths } \\
\hline 1 & Sidewalks too narrow \\
\hline 2 & Absence of sidewalks \\
\hline 3 & Uneven sidewalks \\
\hline 4 & Presence of obstacles on sidewalks \\
\hline 5 & Absence of pedestrian crossing \\
\hline 6 & Faded pedestrian crossing \\
\hline 7 & Incorrect positioning of pedestrian crossing \\
\hline 8 & Absence of ADA ramps on sidewalks \\
\hline 9 & Vehicles parked on the sidewalks \\
\hline 10 & Parked vehicles that obstruct pedestrian crossing \\
\hline 11 & Inadequate drivers' behavior \\
\hline 12 & Damaged road pavement \\
\hline 13 & Roadway too narrow and absence of sidewalks \\
\hline 14 & Absence or inadequacy of street lighting \\
\hline 15 & $\begin{array}{l}\text { Absence or inadequacy of signalized pedestrian } \\
\text { crossings }\end{array}$ \\
\hline 16 & Other \\
\hline
\end{tabular}

Table 2. Variables used for the cluster analysis

\begin{tabular}{|c|c|c|c|c|}
\hline No driving license & No & $66,67 \%$ & Yes & $33,33 \%$ \\
\hline No still driving & No & $48,37 \%$ & Yes & $51,63 \%$ \\
\hline Accidents driving & No & $39,87 \%$ & Yes & $60,13 \%$ \\
\hline $\begin{array}{c}\text { Accidents } \\
\text { pedestrian }\end{array}$ & No & $24,18 \%$ & Yes & $75,82 \%$ \\
\hline Vision problems & No & $52,94 \%$ & Yes & $47,06 \%$ \\
\hline Hearing problems & No & $32,03 \%$ & Yes & $67,97 \%$ \\
\hline Mobility problems & No & $24,18 \%$ & Yes & $75,82 \%$ \\
\hline Gender & Male & $50,98 \%$ & Female & $49,02 \%$ \\
\hline
\end{tabular}

\section{Results and Discussion}

\subsection{K-Means Cluster Analysis}

As shown in Table 3, critical issues of pedestrian paths were grouped in clusters by using SPSS software. To use K-Means clustering, the number of clusters is arbitrarily determined, either from existing knowledge of the data and the approximate number of groups you want to divide the data into. Of course, a good approach to K-Means is to try several numbers of clusters and see which number best represents the data or produces any significant differences in analysis. Different models of clusters were therefore estimated, from one to seven, for selecting the suitable number of clusters. For further analysis, the critical issues of pedestrian paths were divided into five clusters. Table 3 shows the clusters membership.

Table 3. Clusters membership

\begin{tabular}{|c|c|c|}
\hline Critical issues of pedestrian paths & Cluster & Distance \\
\hline 1 & 3 & 0.242 \\
\hline 2 & 2 & 0.000 \\
\hline 3 & 3 & 0.185 \\
\hline 4 & 3 & 0.244 \\
\hline 5 & 5 & 0.286 \\
\hline 6 & 5 & 0.247 \\
\hline 7 & 3 & 0.420 \\
\hline 8 & 5 & 0.365 \\
\hline 9 & 3 & 0.238 \\
\hline 10 & 1 & 0.000 \\
\hline 11 & 5 & 0.327 \\
\hline 12 & 3 & 0.432 \\
\hline 13 & 3 & 0.277 \\
\hline 14 & 4 & 0.000 \\
\hline 15 & 3 & 0.318 \\
\hline 16 & 3 & 0.314 \\
\hline & & \\
\hline
\end{tabular}

The first cluster is composed only by item 10, i.e. "parked vehicles that obstruct pedestrian crossing". This cluster can therefore be named Irregular parking. Cluster 2 is composed only by item 2, i.e. "absence of sidewalks". The second cluster can therefore be named Absence of sidewalks. The third cluster groups together 9 items, i.e. item 1 ("sidewalks too narrow"), item 3 ("uneven sidewalks"), item 4 ("presence of obstacles on sidewalks"), item 7 ("incorrect positioning of pedestrian crossing"), item 9 ("vehicles parked on the sidewalks"), item 12 (“damaged road pavement”), item 13 ("roadway too narrow and absence of sidewalks"), item 15 ("absence or inadequacy of signalized pedestrian crossings"), item 16 ("other"). Cluster 3 can therefore be named Problems of sidewalks and of the correct use of pedestrian crossings. Cluster 4 is composed only by item 14, i.e. "absence or inadequacy of street lighting". The fourth cluster can therefore be named Absence or inadequacy of street lighting. Finally, Cluster 5 groups together 4 items, i.e. item 5 ("absence of pedestrian crossing"), item 6 ("faded pedestrian crossing"), item 8 ("absence of ADA ramps on sidewalks") and item 11 ("inadequate drivers' behavior"). Cluster 5 can therefore be named Problems of pedestrian crossings and of drivers' behavior.

Table 4 shows the ANOVA analysis results and allows to understand which variables affect more the identification of the clusters. The variables mostly 
contributing to the identification of the clusters are Driving license (Sig. $=0.000$ ), Still driving (Sig. $=0.000$ ), Gender (Sig. $=0.000)$, Vision problems (Sig. $=0.002$ ) and Accidents driving (Sig=0.028). Accidents pedestrian ( $\mathrm{Sig}=0.299$ ), Hearing problems $(\mathrm{Sig}=0.117)$ and Mobility problems $($ Sig $=0.115)$ are instead the variables less affecting the division into different clusters.

Table 4. ANOVA analysis results

\begin{tabular}{|c|c|c|c|c|c|c|}
\hline & \multicolumn{2}{|c|}{ Cluster } & \multicolumn{4}{c|}{ Error } \\
\cline { 2 - 7 } & $\begin{array}{c}\text { Mean } \\
\text { Square }\end{array}$ & $\mathrm{df}$ & $\begin{array}{c}\text { Mean } \\
\text { Square }\end{array}$ & $\mathrm{df}$ & $\mathrm{F}$ & Sig. \\
\hline $\begin{array}{c}\text { No driving } \\
\text { license }\end{array}$ & 0.083 & 4 & 0.006 & 11 & 13.109 & $\mathbf{0 . 0 0 0}$ \\
\hline No still driving & 0.137 & 4 & 0.010 & 11 & 13.987 & $\mathbf{0 . 0 0 0}$ \\
\hline $\begin{array}{c}\text { Accidents } \\
\text { driving }\end{array}$ & 0.101 & 4 & 0.024 & 11 & 4.122 & $\mathbf{0 . 0 2 8}$ \\
\hline $\begin{array}{c}\text { Accidents } \\
\text { pedestrian }\end{array}$ & 0.028 & 4 & 0.020 & 11 & 1.393 & 0.299 \\
\hline Vision problems & 0.109 & 4 & 0.012 & 11 & 9.018 & $\mathbf{0 . 0 0 2}$ \\
\hline $\begin{array}{c}\text { Hearing } \\
\text { problems }\end{array}$ & 0.039 & 4 & 0.016 & 11 & 2.363 & 0.117 \\
\hline $\begin{array}{c}\text { Mobility } \\
\text { problems }\end{array}$ & 0.037 & 4 & 0.015 & 11 & 2.381 & 0.115 \\
\hline Gender & 0.148 & 4 & 0.008 & 11 & 19.157 & $\mathbf{0 . 0 0 0}$ \\
\hline
\end{tabular}

The judgment expressed by the elderly on the critical issues of pedestrian paths seems to be significantly linked to gender, to the experience as road users, and to vision problems that compromise the correct perception of the road environment. On the other hand, the least significant variable in conditioning the judgment on critical issues is that associated with road accidents that respondents had pedestrians. Hearing and mobility problems, even if conditions the perception of urban pedestrian paths, are less significant than sight problems. Basically, in identifying the critical issues of pedestrian paths, the elderly are mainly conditioned by the difficulty of correctly seeing the paths themselves and of perceiving the information deriving from the road environment as a whole.

Table 5 shows the profiles of the clusters obtained with the K-Means procedure.

Each group is represented by a center which originate a vector (row) whom components are the means of the values of the variables that defines the coordinates of the objects belonging to that group. The final cluster centers can range from 0 to 1 . The closer the value is to 1 , the closer is the condition "Yes" expressed by the variable (except for the variable Gender for which the closer the value is to 1 the more are women than men). These conditions are all representative of age related declines in perceptual and physical abilities (vision, hearing and mobility problems) or of experiences as road user (no driving license, no still driving, accidents driving, accident pedestrian) which can affect the opinion on the critical issues of pedestrian paths.

The characteristics of the five clusters are given below.

- $\quad$ Cluster 1 (Irregular parking): All respondents of this group are men. Moreover, the majority of respondents belonging to this group had accidents while driving.

- Cluster 2 (Absence of sidewalks): This group is mainly composed by women who don't drive anymore, who never had the driving license and have vision and hearing problems.

- Cluster 3 (Problems of sidewalks and of the correct use of pedestrian crossings): Table 5 shows that no particular characteristics of respondents belonging to this group can be identified. This suggests that respondents who identify these critical issues of pedestrian paths don't have particular characteristics. This also suggests that critical issues associated to cluster 3 are commonly perceived by pedestrians regardless of age related declines in perceptual and physical abilities and regardless of their experiences as road users.

- $\quad$ Cluster 4 (Absence or inadequacy of street lighting): All respondents of this group had accidents while driving and are men.

- Cluster 5 (Problems of pedestrian crossings and of drivers' behavior): Table 5 shows that no particular characteristics of respondents belonging to this group can be identified. This suggests that respondents who identify these critical issues of pedestrian paths don't have particular characteristics. As with cluster 3, the critical issues associated to cluster 5 are commonly perceived by pedestrians regardless of age related declines in perceptual and physical abilities and regardless of their experiences as road users.

Table 5. Final cluster centers

\begin{tabular}{|c|c|c|c|c|c|}
\hline & \multicolumn{5}{|c|}{ Cluster } \\
\cline { 2 - 6 } & 1 & 2 & 3 & 4 & 5 \\
\hline No driving license & 0.00 & $\mathbf{0 . 6 8}$ & 0.29 & 0.00 & 0.21 \\
\hline No still driving & 0.45 & $\mathbf{0 . 9 1}$ & 0.52 & 0.00 & 0.30 \\
\hline Accidents driving & $\mathbf{0 . 8 2}$ & 0.55 & 0.46 & $\mathbf{1 . 0 0}$ & 0.41 \\
\hline Accidents pedestrian & 0.45 & 0.31 & 0.23 & 0.00 & 0.27 \\
\hline Vision problems & 0.73 & $\mathbf{0 . 7 8}$ & 0.62 & 0.40 & 0.28 \\
\hline Hearing problems & 0.27 & $\mathbf{0 . 5 2}$ & 0.25 & 0.00 & 0.18 \\
\hline Mobility problems & 0.45 & 0.30 & 0.13 & 0.00 & 0.22 \\
\hline Gender & $\mathbf{0 . 0 0}$ & $\mathbf{0 . 7 8}$ & 0.50 & $\mathbf{0 . 0 0}$ & 0.29 \\
\hline
\end{tabular}


Table 6 shows the Euclidean distances between the final cluster centers. The higher is this distance, the higher is the difference between groups. It can be seen that the distance between cluster 3 and cluster 5 is the minimum (0.478). That is a confirmation of the fact that cluster 3 and cluster 5 are similar. For both these clusters, indeed, no particular characteristics of respondents were identified.

Table 7 summarizes the "profiles" of the elderly pedestrians identified in relation to the 5 clusters defined by means of the K-Means cluster analysis

Table 6. Distances between final cluster centers

\begin{tabular}{|c|c|c|c|c|c|}
\hline \multicolumn{7}{|c|}{ Cluster } \\
\hline & 1 & 2 & 3 & 4 & 5 \\
\hline 1 & & 1.209 & 0.793 & 0.906 & 0.791 \\
\hline 3 & 1.209 & & 0.725 & 1.644 & 1.109 \\
\hline 4 & 0.793 & 0.725 & & 1.034 & $\mathbf{0 . 4 7 8}$ \\
\hline 5 & 0.906 & 1.644 & 1.034 & & 0.858 \\
\hline
\end{tabular}

Table 7. Profiles of the elderly pedestrians obtained from the K-Means cluster analysis.

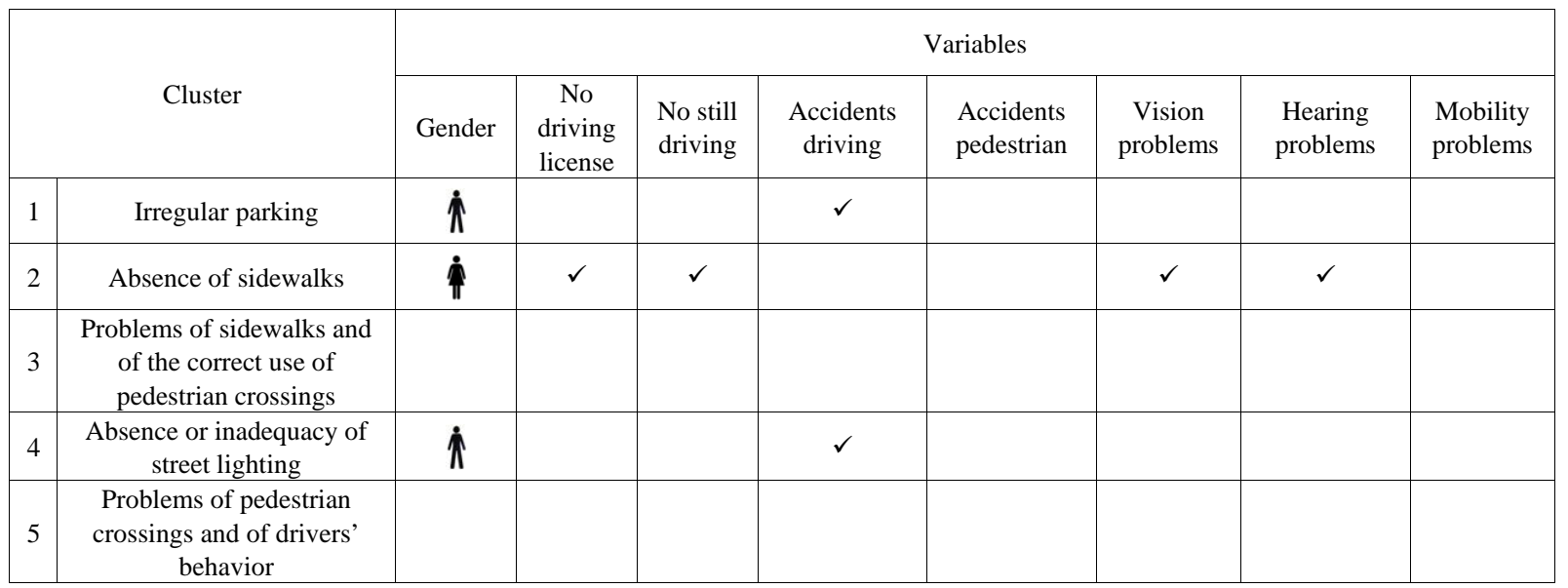

The following considerations can be made:

1) the critical issues concerning the correct use of sidewalks and pedestrian crossings (cluster 3 and cluster 5) are not associated with a specific "profile" of elderly pedestrian. These critical issues, therefore, constitute a safety deficit highlighted in a systematic manner by almost all the sample of users interviewed;

2) also the problems related to aggressive drivers' behavior are highlighted almost indiscriminately by all the respondents;

3) the problem of irregular parking (cluster 1) and the problems related to the absence or the inadequacy of street lighting (cluster 4 ) are mainly indicated by men who had driving accidents during their experience as road users.

4) the "profile" constituted by women who have never driven and who have sight and hearing problems is associated with the problems related to the absence of sidewalks (cluster 2).

\subsection{Hierarchical Cluster Analysis}

Hierarchical clustering allows to confirm the number of clusters which was hypothesized with the K-Means clustering. The optimal number of clusters with the hierarchical method is determined by the minimum number of groups with the maximum amount of distance between group means. Frequently, this is illustrated with a dendrogram of the merging clusters. Using a dendrogram, the ideal number of clusters is determined by the number of clusters intersected when drawing a horizontal line through the largest vertical distance between merging clusters. Similar to K-Means, the optimal value of clusters must be chosen, but this method gives some perspective as to what the ideal value may be.

The hierarchical clustering allowed to illustrate the hierarchical organization of groups as shown in the dendrogram of Figure 1. 


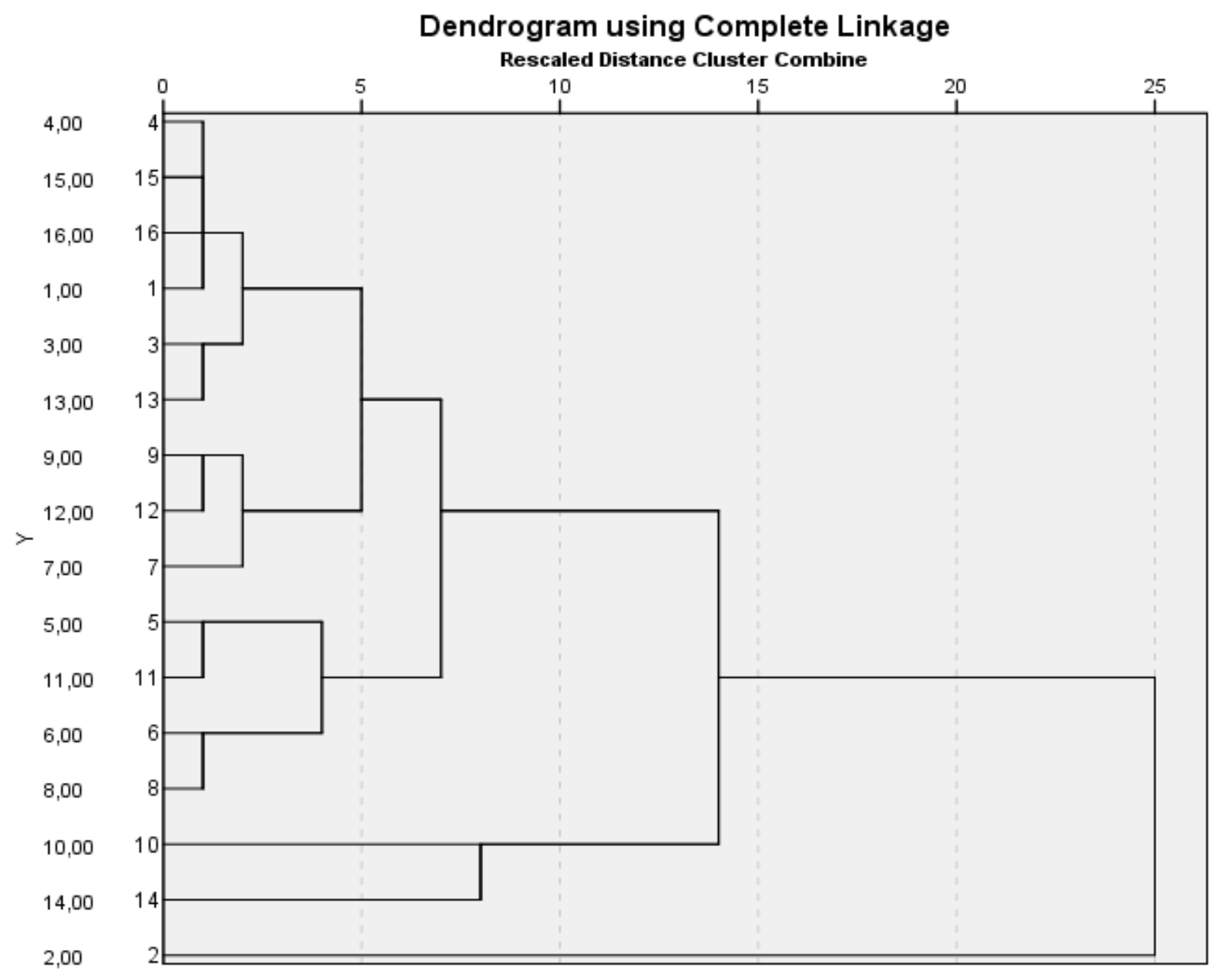

Figure 1. Hypothesis scheme

This visualization confirms the previous result, but offers also a hierarchical view of the clusters. By cutting the dendrogram at height 6 , corresponding to the highest jump between levels of similarity, five clusters homogeneous as for their level of perceived safety are obtained. These clusters correspond to the five clusters resulting from the K-Means cluster analysis. The hypothesis made for K-Means cluster analysis was therefore fully confirmed by hierarchical cluster analysis.

\section{Conclusions}

It is widely recognized that human factors may contribute to accident involvement in traffic [42,43]. The literature on human factors and road user behavior is extensive (e.g. [44, 45]). The understanding of pedestrian behavior in urban contests may assist to improve design and planning of road and traffic environment, and consequently to improve of pedestrian comfort and safety. It has also been shown that road and traffic factors alone may explain only a small part of pedestrian walking and crossing behavior in urban areas [46]. However, human factors related to pedestrians have received less attention in the literature compared to other road users [47].

This study wants therefore to understand how human factors influence elderly pedestrian perception of critical issues of pedestrian paths. The aspects related to human factors considered were the gender, the factors associated with the experience as road users and the factors related to age related problems (mobility, vision and hearing problems). More specifically, the aim was to capture and analyze the key components that influence the elderly pedestrians' perception of pedestrian paths and to identify how these perceptions change for different pedestrian "profiles" based on human factors.

The results of this research show that the judgment expressed by the elderly on the critical issues of pedestrian paths they usually walk is significantly linked to gender, to their experience as road users, and to vision problem, which compromise the correct perception of the road environment. However, cluster analysis allowed to identify few "profiles" of elderly pedestrians with regard to their perception of critical issues of pedestrian paths. These findings should be considered in light of the limitations of the present research. Extending the survey to a larger and more representative sample may reveal additional critical issues and additional "profiles" of elderly pedestrians. 
Moreover, the limits associated to the specific sites considered for the survey have to be considered.

Despite the aforementioned limits, the results have however showed important confirmations regarding the problems of urban pedestrian paths which are a priority for elderly users. The "profiles" identified, although few in number, are indeed perfectly consistent with the different elderly users' characteristics, capabilities and needs that should be considered and prioritized when designing pedestrian safety measures.

Age is connected to a variety of characteristics and skills that influence the risk of pedestrian traffic injury. These age-related characteristics can also affect the way in which people of different ages interact with pedestrian safety measures and require therefore particular attention when planning road safety measures.

Several factors work together to increase the risk of older pedestrians [12, 48]:

- Deterioration in visual acuity may have a negative impact on their ability to cross the road safely. In general, older pedestrians look less at traffic and accept significantly smaller gaps in traffic when crossing the road than younger pedestrians.

- $\quad$ Reduced mobility can render older pedestrians unable to react quickly in imminent danger to avoid a crash.

- Health conditions or frailty can result in greater injury severity when a crash occurs.

- The speed of elderly pedestrians does not itself increase the accident risk: the risk comes from the speed of the traffic and, in particular, from automated signals that do not allow sufficient time for slower pedestrians to cross safely.

The following measures can be implemented to improve the safety of older pedestrians, also contributing to the improvement of environmental quality through the reduction of noise and atmospheric pollutants [48, 49, 50, 51]:

- Increase the time allocated to pedestrians at midblock signalized crosswalks.

- Install midblock high-visibility crosswalks.

- $\quad$ Repair broken sidewalks and pedestrian ramps.

- $\quad$ Replace missing and/or upgrade existing signs.

- Install pedestrian refuge islands or, preferably, raised medians.

- Install traffic-calming measures at streets with high pedestrian flows.

- $\quad$ Reduce interactions between pedestrians and other road user types through the physical separation of flows in urban areas using for example raised planter boxes and outdoor seating and the use of wider sidewalks.

- Improve the conspicuity of pedestrians for drivers.

- Improve the perception of elderly pedestrians about other road users.

At road intersections, roadway design and signalization play a critical role to pedestrian safety. Some interventions that could be implemented are the following [50, 52]:

- Adjusting the traffic signals to allow for the slower walking speed of elderly pedestrians in intersection areas.

- Use of exclusive pedestrian signal phases.

- Use of protected phases during signalization.

- Pedestrian islands or sidewalk extensions in urban areas.

Moreover, roundabouts have a positive impact to safety especially when constructing large splitter islands, banning parking near roundabouts, using adequate street lighting, speed reduction installations, reduced width of circular carriageway, as well as increased deflection and improved signing [36, 39, 41, 50]. The roundabouts also contribute to improving the liveability and pleasantness of the pedestrian paths, giving rise to environmental improvements such as the reduction of atmospheric and acoustic pollution [53].

Out-of-vehicle ITS applications have the potential to enhance speed limit compliance. These applications include dynamic messaging, in the form of active speed warning signs and variable message signs $[48,50]$.

Regarding conspicuity, measures should target $[48,50]$ :

- Lighting at intersections and pedestrian crossings.

- Installation of conspicuous 'give way to pedestrian' signals.

- $\quad$ Reflective pavement markings.

Regarding behavioural factors, for example risk taking behaviours such as the inability to scan and react properly to urban areas and so on, successful countermeasures may include [48, 50]:

- Educational campaigns to promote the use of specialized clothing.

- Educational campaigns to increase the awareness of age-related cognitive and sensory declines.

- Raise public awareness about the safety needs of elderly pedestrians.

- Inform and train pedestrians about the proper behavior to adopt on the road or related to novel traffic rules, situations and road layouts in urban and rural areas

- $\quad$ Reduce legal speed limits.

- Strengthen enforcement of laws on speed limits, and drink-driving.

In a future study, the authors intend to use section 5 of the questionnaire (related to the solutions for critical issues of pedestrian paths proposed by the respondents) to verify which of the actions and strategies indicated above were actually considered a priority by the elderly interviewed.

\section{Acknowledgements}

This work has been partially financed by the University of Catania within the project "Piano della Ricerca 
Dipartimentale 2016-2018” of the Department of Civil Engineering and Architecture.

\section{REFERENCES}

[1] K.J. Foreman, N. Marquez, A. Dolgert, K. Fukutaki, M. Mcgaughey, M.A. Pletcher et al. Forecasting life expectancy, years of life lost, all-cause and cause-specific mortality for 250 causes of death: reference and alternative scenarios 2016-2040 for 195 countries and territories. Lancet, 2018.

[2] World Health Organization. Road Traffic Injuries-Fact Sheet.https://www.who.int/en/news-room/fact-sheets/detai 1/road-traffic-injuries, 2018.

[3] A.V. Moudon, L. Lin, J. Jiao, P. Hurvitz, P. Reeves. The risk of pedestrian injury and fatality in collisions with motor vehicles, a social ecological study of state routes and city streets in king country. Accid. Anal. Prev. 43, 11-24, 2011.

[4] European Commission. Traffic Safety Basic Facts on Pedestrians; European Commission, Directorate General for Transport; Available online: https://ec.europa.eu/transp ort/road_safety/sites/roadsafety/files/pdf/statistics/dacota/b fs20xx_pedestrians.pdf, 2018

[5] H.W. Kohl, C.L. Craig, E.V. Lambert, S. Inoue, J.R. Alkandari, G. Leetongin, S. Kahlmeier. The pandemic of physical inactivity: global action for public health. The Lancet, 380(9838), 294-305, 2012. https://doi.org/10.1016 /S0140-6736(12)60898-8.

[6] A. Dommes, V. Cavallo. The role of perceptual, cognitive, and motor abilities in street-crossing decisions of young and older pedestrians. Ophthalmic and Physiological Optics, 31, 292-301, 2011.

[7] A. Dommes, V. Cavallo, J.A. Oxley. Functional declines as predictors of risky street-crossing decisions in older pedestrians. Accident Analysis and Prevention, 59, 135143, 2013.

[8] G. Dunbar, C.A. Holland, E.A. Maylor. Older pedestrians: A review. London: Department for Transport, 2004.

[9] Observatoire National Interministériel de Sécurité Routiere (ONISR). La sécurité routière en France. Bilan de l'année 2011. Paris: La documentation Française, 2011.

[10] J.A. Oxley, B.N. Fildes, E. Ihsen, J.L. Charlton, R.H. Day. Differences in traffic judgements between young and old adult pedestrians. Accident Analysis \& Prevention, 29, 839-847, 1997.

[11] H. Fontaine, Y. Gourlet. Fatal pedestrian accident in France: A typological analysis. Accident Analysis and Prevention, 29, 303-312, 1997.

[12] J. Garrard. Senior Victorians and walking: Obstacles and opportunities. Summary Report. Melbourne: Victoria Walks, 2013.

[13] SHARE: The Survey of Health, Ageing and Retirement in Europe-2011.
[14] N. Distefano, S. Leonardi, G. Pulvirenti, Home-school travel: Analysis of factors affecting Italian parents' mode choice. Civil engineering and Architecture, Vol. 7, N. 3, Pages 75-87, 2019. DOI: 10.13189/cea.2019.070302

[15] S. O’Hern, J. Oxley. Understanding travel patterns to support safe active transport for older adults. Journal of Transport and Health, 2 (1) 79-85 doi: 10.1016/j.jth.2014. 09.016, 2015.

[16] F.C. Bijnen,C.J. Caspersen, E.J. Feskens, W.H. Saris, W.L. Mosterd, D. Kromhout. Physical activity and 10-year mortality from cardiovascular diseases and all causes: the Zutphen Elderly Study. Archives of Internal Medicine, 158(14), 1499-1505, 1998.

[17] R.V. Luepker, D.M. Murray, D.R. Jacobs Jr, M.B. Mittelmark, N. Bracht, R. Carlaw, R. Crow, P. Elmer, A. Finnegan, A.R. Folsom. Community education for cardiovascular disease prevention: risk factor changes in the Minnesota Heart Health Program. American journal of public health, 84(9), 1383-1393, 1994.

[18] J. Oxley, J. Charlton, B. Corben, B. Fildes. Crash and injury risk of older pedestrians and identification of measures to meet their mobility and safety needs. The 7th International Conference on Walking and Liveable Communities. Victoria: Monash University, 2006.

[19] J. Kerr, D. Rosenberg, L. Frank. The role of the built environment in healthy aging: Community design, physical activity, and health among older adults. J. Plan. Lit., 27, 4360, 2012.

[20] T. Sugiyama, C.W. Thompson. Outdoor environments, activity and the well-being of older people: Conceptualising environmental support. Environ. Plan. A, 39, 1943-1960, 2007.

[21] Start Active, Stay Active: A Report on Physical Activity from the Four Home Countries' Chief Medical Officers, 2011.

[22] B.E. Saelens, S.L. Handy. Built environment correlates of walking: A review. Med. Sci. Sports Exerc., 40, S550-S566, 2008.

[23] A. Loukaitou-Sideris. Is it safe to walk? Neighborhood safety and security considerations and their effects on walking. J. Plan. Lit., 20, 219-232, 2006.

[24] K. Ball, A. Bauman, E. Leslie, N. Owen. Perceived environmental aesthetics and convenience and company are associated with walking for exercise among Australian adults. Prev. Med., 33, 434-440, 2001.

[25] P.M. Hess, A.V. Moudon, M. Snyder, K. Stanilov. Site design and pedestrian travel. Transp. Res. Rec., 9-19, 1999.

[26] C. Lee, A.V. Moudon. Neighbourhood design and physical activity. Build. Res. Inf., 36, 395-411, 2008.

[27] D.F. Lawrence, O.E. Peter. The built environment and human activity patterns: Exploring the impacts of urban form on public health. J. Plan. Lit., 2001.

[28] G.O. Cunningham, Y.L. Michael. Concepts guiding the study of the impact of the built environment on physical activity for older adults: A review of literature. Am. J. Health Promot., 18, 435-443, 2004. 
[29] Y.L. Michael, M.K. Green, S.A. Farquhar. Neighborhood design and active aging. Health Place, 12, 734-740, 2006.

[30] D. Lockett, A. Willis, N. Edwards. Through seniors' eyes: An exploratory qualitative study to identify environmental barriers to and facilitators of walking. Can. J. Nurs. Res., 37, 48-65, 2005.

[31] R. Zandieh, J. Martinez, J. Flacke, P. Jones, M. van Maarseveen. Older Adults' Outdoor Walking: Inequalities in Neighbourhood Safety, Pedestrian Infrastructure and Aesthetics. International Journal of Environmental Research and Public Health. Vol. 13 (12), Article n. 1179, 2016.

[32] D. Kahlert, W. Schlicht. Older People's Perceptions of Pedestrian Friendliness and Traffic Safety: An Experiment Using Computer-Simulated Walking Environments. International Journal of Environmental Research and Public Health. Vol. 12 (8), 10066-10078, 2016.

[33] S. Lord, M.-S. Cloutier, B. Garnier, Z. Christoforou. Crossing road intersections in old age - With or without risks? Perceptions of risk and crossing behaviours among the elderly. Transportation Research Part F: Traffic Psychology and Behaviour. Vol. 55, 282-296, 2018.

[34] Y. Noh, M. Kim, Y. Yoon. Elderly pedestrian safety in a rapidly aging society-Commonality and diversity between the younger-old and older-old. Traffic Injury Prevention. Vol. 19 (8), 874-879, 2018.

[35] J. Van Cauwenberg, V. van Holle, D. Simons, R. Deridder, P. Clarys, L. Goubert, J. Nasar, J. Salmon, I. de Bourdeaudhuij, B. Deforche. Environmental factors influencing older adults' walking for transportation: a study using walk-along interviews. International Journal of Behavioral Nutrition and Physical Activity. Vol. 9, Article n. 85, 2012.

[36] N. Distefano, S. Leonardi, G. Pulvirenti. Factors with the greatest influence on drivers' judgment of roundabouts safety. An analysis based on web survey in Italy. IATSS Research 42, 265-273, 2018. DOI: 10.1016/j.iatssr.2018.0 4.002

[37] N. Giuffrida, M. Ignaccolo, G. Inturri. Using multiple correspondence analysis to improve safety in interaction between road transit and public spaces. In Town and Infrastructure Planning for Safety and Urban Quality: Proceedings of the XXIII International Conference on Living and Walking in Cities (LWC 2017), June 15-16, 2017, Brescia, Italy (p. 129). CRC Press, 2018.

[38] N. Distefano, S. Leonardi, F. Consoli. Drivers’ Preferences for Road Roundabouts: A Study based on Stated Preference Survey in Italy. KSCE Journal of Civil Engineering, Vol. 23 (11), no. 2016, pp. 1-11, 2019. DOI: 10.1007/s12205-019$1363-9$

[39] S. Leonardi, N. Distefano, G. Pulvirenti. Italians' public opinion on road roundabouts: A web based survey. European Transportation Research Procedia, IN PRESS.

[40] M. Ignaccolo, G. Inturri, N. Giuffrida, M. Le Pira, V. Torrisi. Public Engagement for Designing New Transport Services: Investigating Citizen Preferences from a Multiple Criteria Perspective. Transportation Research Procedia, 37, 91-98, 2019.

[41] S. Leonardi, N. Distefano, G. Pulvirenti. Multiple
Correspondence Analysis (MCA) for the evaluation of risk perception of roundabouts for young people. European Transport \Trasporti Europei, Issue 72, Paper $n^{\circ} 4$, ISSN 1825-3997, 2019.

[42] S. Birth. Human factors for safer road infrastructure, Routes-Roads, pp. 30-39, 2013.

[43] A. Borsos, S. Birth, H. Vollpracht. The role of human factors in road design. 6th IEEE International Conference on Cognitive Infocommunications (CogInfoCom), Gyor, pp. 363-367, 2015.

[44] N. Distefano, S. Leonardi, G. Pulvirenti, R. Romano, N. Merat, E. Boer, E. Woolridge. Physiological and driving behavior changes associated to different road intersections. European Transport $\backslash$ Trasporti Europei, IN PRESS.

[45] R. Fuller, J.A. Santos. Human Factors for Highway Engineers. Elsevier Science, UK, 2002.

[46] E. Papadimitriou, G. Yannis, J. Golias. A critical assessment of pedestrian behaviour models. Transportation Research Part F Vol. 12 (3), pp. 242-255, 2009.

[47] E. Papadimitriou, S. Lassarre, G. Yannis. Human factors of pedestrian walking and crossing behaviour. Transportation Research Procedia 25C pp. 2007-2020, 2017.

[48] World Health Organization. Pedestrian safety. A road safety manual for decision-makers and practitioners. https://www.who.int/roadsafety/projects/manuals/pedestria n/en/, 2013.

[49] N. Distefano, S. Leonardi. Evaluation of the benefits of traffic calming on vehicle speed reduction. Civil engineering and Architecture, Vol. 7, N. 4, Pages 200-2014, 2019. DOI: $10.13189 /$ cea.2019.070403

[50] E. Polders, T. Brijs, E. Vlahogianni, E. Papadimitriou, G. Yannis, F. Leopold, C. Durso, K. Diamandouros. ElderSafe - Risks and countermeasures for road traffic of the elderly in Europe - Final report, $\mathrm{N}^{\circ}$ MOVE/C4/2014-244. https://ec.europa.eu/transport/road_safety/sites/roadsafety/f iles/pdf/studies/eldersafe_final_report.pdf, 2015.

[51] N. Distefano, S. Leonardi. Experimental investigation of the effect of speed bumps in sequence on noise emission level from motor vehicles. Noise Control Engr. J. 63 (6), November-December 2015. DOI: 10.3397/1/376352

[52] S. Canale, N. Distefano, S. Leonardi. Comparative analysis of pedestrian accidents risk at unsignalized intersections. The Baltic Journal of Road and Bridge Engineering, 2015, vol.10, n.4. DOI: 10.3846/bjrbe.2015.36

[53] N. Distefano, S. Leonardi. Experimental investigation of the effect of roundabouts on noise emission. Noise Control Engr. J. 67 (4), July-August 2019. Pages 282-294. https://doi.org/10.3397/1/376725 